\title{
MAURICE BEDOT
}

1859-1927

PA R

\section{P. REVILLIOD}

Retracer la carrière scientifique et administrative de Maurice Bedot c'est passer en revue plus de quarante années d'une activité féconde et inlassable mise au service de sa ville, de son pays et de la science, c'est aussi se remémorer presqu'un demi-siècle de vie scientifique genevoise.

Maurice Bedot n'a pas voulu que les dons remarquables qui lui avaient été dispensés fussent réservés à la seule recherche scientifique, et employés à satisfaire seulement son désir de s'instruire; il les utilisa en premier lieu à remplir les charges qui lui avaient été confiées comme celles qu'il s'était imposées, pour le plus grand profit des institutions dont il avait la direction, et des naturalistes de son pays dont il a toujours cherché à seconder les efforts. En effet, rien de ce qui touchait au développement et à la mise en valeur de nos richesses scientifiques ne le laissait indifférent et dans tous les domaines de la vie intellectuelle de notre cité, on retrouve les traces de son dévouement et de son activité. Pour caractériser l'œuvre si diverse et si désintéressée de Maurice Bedot nous rappellerons en trois paragraphes distincts ce que fut la carrière du zoologiste, du directeur du Muséum d'histoire naturelle de Genève et du fondateur de la Revue suisse de zoologie.

\section{Les études, les publications.}

Maurice Bedot était né à Genève, en 1859. Passant l'été à Satigny où son père avait une propriété dans ce beau pays du Mandement, le goût pour l'histoire naturelle, le sens de l'observation et la passion des collections se développèrent

Rev. Suisse de Zool. 'T. 35. 1928. 
de très bonne heure chez lui. Il entra à l'Université à l'époque où deux maitres illustres enseignaient la biologie, Carl Vogt et Hermann Fol. L'émulation était grande alors dans les laboratoires du sous-sol de l'Université; Vogt dirigeait ses élèves de préférence vers les études d'anatomie comparée et de zoologie systématique. Titulaire d'une chaire d'embryogénie comparée, Fol défrichait des terrains nouveaux, initiant ses disciples dans l'embryogénie et la physiologie des Invertébrés, et des Vertébrés, dans l'organisation des Cœlentérés, dans la cytologie, etc.

Maurice Bedot fut enthousiasmé par les leçons d'Hermann Fol, remarquables par la clarté de l'exposition et la nouveauté des idées qu'il y développait. Mais il sut dès les premiers semestres, sortir de Genève et augmenter ses connaissances dans les stations et les laboratoires des pays voisins tout en restant en contact permanent avec son maître. C'est ainsi que la première note qu'il publia en 1882, fut une liste des Siphonophores qu'il avait récoltés dans le golfe de Naples pendant un séjour de cinq mois qu'il fit à la station zoologique de Naples.

Puis il se rendit à Iena où il commença sous la direction d'Oscar Hertwig des recherches sur l'origine et le développement des nerfs spinaux chez les Tritons, travail qu'il termina dans le laboratoire d'Hermann Fol et qu'il présenta comme thèse de doctorat.

Cependant, la station zoologique que Fol avait créée à Villefranche-sur-Mer, faisait connaitre la richesse de la faune qui s'abrite dans cette baie. Maurice Bedot, comme les autres élèves de Fol, y fit de fréquents séjours et de fructueuses pêches. Les abondants matériaux récoltés par Fol étaient généreusement mis à la disposition de ses disciples.

Ces circonstances orientèrent dès lors, Maurice Bedot, vers l'étude des Colentérés.

Les connaissances que l'on avait à cette époque sur les Siphonophores en particulier étaient encore très fragmentaires, et beaucoup de sujets de travaux sur la conformation et la signification de leurs organes, sur leur développement et leur systématique s'offraient à la sagacité des naturalistes. Hæckel publiait à ce moment son essai de classification des Siphonophores. Bedot apporta à son tour d'importantes contributions à l'étude de ces animaux par des recherches sur les Vélelles, les Physalies, les Agalmidés, les Forskalies, etc. 
C'est au laboratoire de Villefranche que Maurice Bedot se lia avec Camille Pictet d'une profonde amitié. Les deux jeunes naturalistes décidèrent d'entreprendre un voyage d'études dans l'archipel malais. Leur but était de visiter les principales îles de l'archipel et d'étudier la faune marine en un point propice qu'il s'agissait de trouver. IIs s'embarquèrent le 9 février 1890.

Après avoir visité un certain nombre d'îles, en récoltant en passant des spécimens de la faune terrestre, s'arrêtant un peu plus longtemps à Bornéo pour explorer la province de Sarawak, ils résolurent de se fixer à Amboine, dont la baie était déjà célèbre pour ses "jardins sous-marins", formés de Madrépores aux couleurs variées et abritant une faune symbiotique fort intéressante.

Se livrant à la pêche dès l'aube jusqu'à 9 heures du matin, passant aux travaux de laboratoire jusqu'à 4 heures pour pêcher de nouveau jusqu'au coucher du soleil, les deux zoologistes genevois s'astreignirent à ce rude labeur pendant deux mois; grâce à leur méthode de travail et à leur ardeur, ils firent, durant ce laps de temps relativement court, une récolte exceptionnellement riche. Pour citer un exemple, nous rappellerons qu'en se donnant la peine de casser à coups de hache des blocs de vieux Madrépores et de Tubipores ils découvrirent plus de 90 espèces de petits Crustacés qui s'y trouvaient réfugiés et dont 21 décrites plus tard par L. Zehntner étaient nouvelles pour la science. L'étude et la détermination de la belle collection rapportée par Bedot et Pictet exigèrent la collaboration d'une trentaine de naturalistes dont les travaux forment deux gros volumes.

Tandis que Camille Pictet décrivait les Hydraires, Bedot se réserva l'étude des Siphonophores et celle des Madréporaires. Cette dernière, comprenant la description de 64 espèces et variétés, est illustrée par une quarantaine de très belles planches. Si la faune recueillie soit sur le fond, dans les jardins de Madrépores, soit le long des rives de la baie d'Amboine, comprenant par conséquent les animaux néritiques et nectoniques, révéla tant de formes différentes de celles de nos mers tempérées, ou encore inconnues, il n'en fut pas de même pour les organismes formant le plancton proprement dit de la baie, soit les animaux eupélagiques.

L'étude de cette faune et sa comparaison avec des animaux. eupélagiques de régions tempérées ou boréales font le sujet d'un travail très intéressant aux conclusions nouvelles pour l'époque. 
Appuyé sur les résultats obtenus dans son étude sur les Siphonophores, ainsi que sur ceux d'autres groupes composés d'animaux uniquement pélagiques (Salpes, Chétognathes, Méduses, Ptéropodes, etc.). Bedot montre que le $80 \%$ des espèces de la baie d'Amboine a été trouvé aussi dans un autre océan, et le $72 \%$ commun à l'Atlantique et au Pacifique. Les régions bionomiques que l'on a cru pouvoir établir pour les animaux pélagiques n'existent pas en réalité; les différences que l'on a observées entre des faunes de régions diverses sont dues à notre ignorance de la variation de la faune pélagique selon les saisons, de la faune pélagique profonde et de sa dissémination par les courants.

Deux ans après leur retour, Camille Pictet qui avait été chargé de l'examen des Hydraires des campagnes de l'Hirondelle, décédait après une courte maladie. Ce triste événement qui affecta beaucoup son ami et compagnon d'études et de voyage et privait Genève d'un zoologiste distingué auquel on pouvait prédire un brillant avenir, paraît avoir été pour Bedot le point de départ d'une nouvelle orientation. Il fut prié de reprendre l'étude des Hydraires des campagnes de l'Hirondelle laissée inachevée par Pictet et dès lors, nous le voyons définitivement consacré à des travaux sur les Hydroïdes. Les nouvelles campagnes du yacht "Princesse-Alice », ainsi que la station de Roscoff, entre autres, fournissent de riches récoltes lui permettant de publier une série de travaux sur les Nemertésies, les Antenella, les Aglaophenia, la systématique des Plumularides, etc.

Nous ne voulons pas terminer cette nomenclature sans avoir mentionné l'œuvre de bibliographie intitulée: Matériaux pour servir à l'histoire des Hydroüdes. Elle fut commencée en 1901, et sept parties ont paru jusqu'à aujourd'hui. Chaque partie renferme les travaux publiés pendant une dizaine d'années et comprend un index bibliographique, un état des genres et des espèces et un index de ceux-ci.

Si l'on songe combien l'établissement de la synonymie de ces animaux est hérịssée de difficultés par le fait que beaucoup d'espèces de polypes ont été décrites, sans que la forme méduse fut connue, où d'après les caractères non pas du polype mais de la colonie, qui peuvent varier au cours de son existence, on pourra mesurer facilement les services inappréciables rendus aux spécialistes par cet ouvrage. 
Au cours des études sur les Hydroïdes et les Siphonophores dont les colonies sont parfois si compliquées, chez lesquels la division du travail est poussée si loin, Maurice Bedot fut amené tout naturellement à examiner de près les notions fondamentales de l'individu, de la colonie, de l'espèce et de la société. La recherche de la définition exacte de ces termes en biologie a suscité de grandes controverses. Les résultats de ses méditations sont consignés dans l'Essai sur l'évolution du règne animal et la formation de la société, petit ouvrage de vulgarisation intéressant par l'originalité de ses vues et parfaitement clair dans l'exposé des difficultés que l'on rencontre en cherchant à comparer la société humaine formée d'individus qui ne peuvent plus vivre indépendamment d'elle, et les divers types d'associations que l'on peut observer dans toute la série animale.

De ces notions ainsi que des vastes connaissances qu'il avait acquises sur les questions de bionomie et de zoo-géographie, Maurice Bedot fit profiter de nombreux étudiants. Il avait été nommé en effet professeur extraordinaire de zoologie générale à l'Université de Genève en 1895; il conserva cet enseignement jusqu'en 1912.

Nous citerons encore pour terminer cette revue de l'œuvre scientifique de Maurice Bedot, les recherches poursuivies dans un tout autre domaine sur le type anthropologique des habitants du Valais. En assistant aux opérations de recrutement dans le canton du Valais durant quelques années, il put prendre des mesures sur environ 1200 individus et fournir ainsi des documents précieux à l'étude des brachycéphales des vallées méridionales de ce canton, des sous-dolichocéphales de la vallée du Rhône et spécialernent de ceux des environs de Savièze.

\section{Le Muséum d'histoire naturelle de Genève.}

Le bâtiment actuel du Muséum d'histoire naturelle avait été inauguré en 1872. Depuis l'époque de la fondation du Musée académique en 1811, les collections fort disparates qui le composaient, n'avaient cessé de s'accroître; il était devenu nécessaire de grouper en un musée d'histoire naturelle celles de zoologie, de paléontologie, de géologie et de minéralogie.

La Commission chargée d'administrer le Musée ne pouvant 
bientôt plus suffire à cette tâche, le Conseil administratif décida en 1878 de nommer un directeur et appela à ce poste Monsieur Godefroy Lunel. Les dons de collections très importantes continuèrent à affluer et des échanges avantageux contribuèrent à l'enrichissement du Musée. Cette période si florissante s'explique par la présence à Genève de nombreux savants qui donnaient à la Ville les riches collections, rassemblées au cours de leurs travaux.

L'accumulation de tous ces trésors devait à bref délai poser à la direction du Musée et aux autorités responsables de graves problèmes d'ordre administratif. L'installation de collections considérables telles que celle d'Insectes de Melly, de Mollusques de Bourguignat, etc., leur intercalation, leur mise en valeur de façon qu'elles offrissent le rendement scientifique le plus grand, exigeaient de nouveaux locaux et un personnel scientifique plus nombreux.

Godefroy Lunel qui assumait la direction du Musée depuis 13 ans, décédait après une courte maladie en novembre 1891 et le mois suivant, Maurice Bedot était appelé aux fonctions de Directeur.

Les autorités municipales plaçaient leur confiance dans ce jeune savant de 32 ans parce qu'elles l'avaient vu à l'œuvre dans quelques occasions déjà, notamment lorsqu'il avait été chargé par la commission d'aller à Paris prendre possession de la collection et de la bibliothèque Bourguignat et de les ramener à Genève, mission délicate dont il s'était fort bien tiré.

Devant cette grande tâche d'organisation, Maurice Bedot se mit aussitôt à l'ouvrage avec cet esprit de décision, cette netteté de vues, cette méthode qui se développèrent encore par la suite. Il lui fallut faire preuve de beaucoup d'énergie et de persévérance pour convaincre les Conseils de la Ville de la nécessité des sacrifices qu'il s'agissait de s'imposer pour faire aux magnifiques collections que Genève possédait, un cadre digne d'elles.

Si l'on consulte les rapports sur l'administration du Musée pendant les premières années d'activité de Maurice Bedot, on voit que les plus sérieux efforts du Directeur comme la plus grande partie des ressources financières furent consacrés à l'organisation des dépôts qui encombraient les sous-sol du Musée, à la transformation de divers locaux et de caves pour gagner de la place ainsi qu'au classement des collections. Un personnel scientifique indispensable pour mener à bien ces travaux manquait à notre adminis- 
tration; mais à cette époque encore, comme un demi-siècle auparavant, la Ville pouvait compter sur une pléiade de bonnes volontés, et le jeune directeur trouva parmi les illustres naturalistes, comme chez les amateurs avertis qui utilisaient nos collections, des auxiliaires désintéressés qui ne craignaient pas de s'astreindre aux besognes les plus modestes. Henri de Saussure, Perceval de Loriol, Auguste Brot, Victor Fatio, pour ne citer que les plus connus de nos savants concitoyens, furent par leur zèle, leur expérience et leur dévouement les précieux collaborateurs des premières années.

Les travaux préliminaires de déblaiement et de mise en ordre bien avancés ou terminés, Maurice Bedot se mit alors à organiser, avec une rigoureuse méthode et selon un plan parfaitement établi, les collections exposées au public. L'idée servant de base à cette organisation nouvelle est que le Musée d'histoire naturelle doit servir à l'instruction générale du public.

Il s'agit pour cela de faire figurer dans les galeries des représentants de tous les groupes d'animaux des plus simples aux plus évolués, mais il faut, d'autre part, éviter d'accumuler un trop grand nombre de spécimens dans les vitrines et n'exposer que les plus caractéristiques, accompagnés d'explications. Le public ne doit pas quitter le Musée en ne gardant que le souvenir d'un chaos d'objets dont il ignore tout, mais au contraire, satisfait d'avoir acquis quelques notions précises et renouvelant son désir de s'instruire.

Ces idées étaient peu répandues à cette époque, et l'on ne ne cherchait encore dans beaucoup de musées qu'à accumuler le plus possible d'objets dans les vitrines pour montrer au public la richesse des collections et l'on n'exposait que certaines catégories d'animaux capables d'attirer facilement son attention.

$\mathrm{Si}$, en Amérique, de grands efforts étaient tentés pour organiser les galeries destinées au public, selon les principes énoncés plus haut, Bedot fut un des premiers en Europe à faire du Musée d'histoire naturelle un établissement pédagogique auxiliaire de l'enseignement public à tous les degrés. Il a exposé ses idées sur l'organisation des musées dans une brochure parue en 1912: Les musées, leur utilité et leurs défauts - Genève, Georg.

Il créa des collections de zoologie générale destinées à orienter le public sur la morphologie, le développement des animaux, les 
conditions d'existence, la variation, etc., et multiplia dans les collections systématiques les cartes de répartition géographique des animaux exposés, les signes indiquant leur genre de vie, les dessins et les schémas montrant leur organisation.

Un musée bien organisé appelle les donateurs; de belles et nombreuses collections entrèrent encore au Musée d'histoire naturelle de Genève. Maurice Bedot ne voyait pas sans angoisse, approcher le moment où la place disponible pour loger tous ces trésors deviendrait tout à fait insuffisante. Dès 1902, il signale cet inconvénient dans les rapports administratifs de chaque année. Sa voix est enfin entendue. Une conférence de délégués du Conseil d'Etat et du Conseil administratif se réunit en septembre 1908 pour étudier la question du transfert du Muséum. L'Etat s'engage à racheter le bâtiment actuel et à remettre à la Ville un terrain à la rue Sturm pour y construire un nouvel édifice.Cette proposition est discutée à diverses reprises dans les Conseils de la Ville pendant les années qui suivent. Le projet de construction du Muséum adopté par le Conseil municipal en 1912 est soumis au referendum et repoussé par les électeurs. L'année suivante, une nouvelle proposition est acceptée par le Conseil municipal et une tentative de referendum échoue. Les travaux de terrassement sont entrepris sur l'esplanade de la rue Sturm, lorsque la guerre éclate. Les travaux sont suspendus; toutes les propositions, soit de les recommencer, soit de modifier les conditions du contrat entre l'Etat et la Ville ou de reprendre les projets sur une base plus modeste, se heurtent contre la nécessité absolue de faire des économies.

Maurice Bedot avait entrepris l'étude de l'installation du nouveau Musée avec un intérêt passionné; il en avait étudié le plan et toutes les questions se rapportant à l'aménagement des collections, à leur exposition, etc., avec une grande minutie; bien que très au courant de l'organisation des musées à l'étranger, il avait fait un voyage en Allemagne, en Autriche et en Hongrie, accompagné d'un de ses assistants, M. J. Favre, dans le but de visiter les principaux musées de ces pays.

Les projets si bien établis, les beaux rêves caressés depuis si longtemps s'évanouissaient au moment où commençait leur réalisation. Il est facile de comprendre combien grands et douloureux durent être les regrets et la déception de celui qui avait voué des. 
années de travail consciencieux à l'étude de cette œuvre qui devait couronner sa carrière.

Bien que déçu et fatigué, et souffrant des premières atteintes de la maladie qui devait l'emporter, Maurice Bedot ne se tint pas dans une attitude purement négative, il chercha, au cours de ces dernières années, à continuer, malgré le manque de place, à développer les collections didactiques et à obtenir des locaux pour y installer les collections ne pouvant être logées au Muséum.

\section{La Revue suisse de zoologie.}

Si Maurice Bedot voua une grande partie de son temps et de ses efforts à l'organisation des collections destinées au public, estimant que le rôle didactique du Musée, instrument de vulgarisation de premier ordre, devait être l'objet de sa plus grande sollicitude, il ne négligea pas pour cela les collections scientifiques.

Les collections genevoises d'entomologie, de malacologie, d'Echinodermes, de Cœlentérés entre autres comptent parmi les plus importantes d'Europe. Elles devaient nécessairement donner naissance à de multiples mémoires et comme on l'a vu, les savants capables de les faire valoir étaient nombreux, dans notre ville, à cette époque. Les bonnes volontés, les talents comme les matériaux d'étude ne manquaient donc pas, mais c'est un périodique national qui faisait défaut.

Dix ans auparavant, Hermann Fol en fondant le Recueil zoologique suisse proclamait que son but était d'empêcher la dispersion des publications des zoologistes suisses dans les périodiques étrangers, de grouper les travaux de nos savants dans une revue consacrée à la zoologie et de faire mieux connaître l'activité scientifique de notre pays.

Le Recueil zoologique suisse ne parut malheureusement que pendant cinq années et fut interrompu lors de la disparition tragique de son fondateur.

Maurice Bedot, attristé par le déficit très sérieux que représentait pour les zoologistes suisses l'absence de périodique, était très désireux de reprendre l'idée de son maitre et de faire revivre le Recueil zỏologique suisse. Aussi, la proposition de créer une revue de zoologie fut elle une des premières qu'il présenta à la Commission 
du Musée quelques mois après sa nomination. Son projet fut accepté. Maurice Bedot s'engageait à diriger la publication et à supporter une partie du déficit, le Musée prenait aussi sa part du déficit possible et acceptait pour sa bibliothèque les publications étrangères qui pourraient ètre obtenues par échange. C'est en juin 1893 que sortait de presse le premier fascicule de la Reoue suisse de zoologie et annales du Musée d'histoire naturelle de Genève.

Dès les premières années, nous voyons la Revue atteindre exactement le but que lui avait fixé son directeur, réunir les publications des naturalistes suisses sur toutes les branches de la zoologie, tout en accueillant aussi des mémoires de valeur de savants étrangers. La Revue est largement ouverte en particulier aux jeunes zoologistes, élèves de nos Universités; le directeur reçoit leurs thèses et cherche à les publier dans les meilleures conditions. On ne saurait assez répéter avec quel désintéressement et avec quelle générosité, Maurice Bedot s'est employé à rendre cet immense service aux jeunes; les chiffres à cet égard sont éloquents; au cours des 34 années d'existence de la Revue, soixante-douze thèses de doctorat ont été imprimées, soit 25 de l'Université de Genève, 21 de l'Université de Bâle, 9 de celle de Berne, 8 de celle de Lausanne, 6 de celle de Zurich et 3 de celle de Neuchâtel. Et nous sommes certains que nombreux sont les zoologistes qui garderont à Maurice Bedot une grande reconnaissance pour la franchise avec laquelle il a su leur indiquer les faiblesses de leur style, et pour la tenacité qu'il a mise à exiger d'eux une parfaite mise au point de leur manuscrit; tel jeune auteur qui, dans sa vanité, aura été impatienté à l'époque où il affrontait les critiques du directeur de la Revue, aura reconnu plus tard le service précieux qui lui avait été rendu et dont il a su profiter au cours de sa carrière.

De nombreux travaux ont fait connaître les riches collections du Muséum de Genève, en particulier les remarquables études de Henri de Saussure sur les Orthoptères, de de Loriol sur les Echinodermes, celles de J. Carl, de Lessert, Peracca, T. Regan, Griffini, etc. Les contributions de J. Roux, G. Bollinger, F. Baumann, etc., ont eu pour objet les collections des Musées de Bâle et de Berne. Il faudrait citer encore tous les mémoires ayant trait à la faune suisse et ceux d'éminents naturalistes étrangers parmi 
lesquels nous voyons les noms de A. Locard, E. Simon, J. Barrois, R. Kœhler et ceux des collaborateurs à l'étude des matériaux rapportés d'Amboine par Bedot et Pictet.

Maurice Bedot accueillait sans parti-pris des études sur les sujets les plus variés, et si les travaux de faunistique dominent, ceux d'anatomie, d'embryologie et de biologie sont aussi nombreux. En parcourant les titres des mémoires et en feuilletant les volumes les uns après les autres, on peut constater l'évolution que subit la zoologie et l'importance que prennent maintenant les recherches d'ordre expérimental.

On comprendra facilement, après l'énumération qui vient d'être faite, que la Revue suisse de zoologie ne tarda pas à jouir d'une juste renommée, mais elle la dut pour une grande part, à sa parfaite tenue. Maurice Bedot ne ménagea ni son temps, ni sa santé surveillant lui-même, jusque dans les plus petits détails, l'impression des travaux qui lui étaient confiés, relisant toutes les épreuves et ne donnant le "bon à tirer " qu'après la revision la plus minutieuse du texte. Homme de goût, il tenait à ce que les travaux paraissant dans sa Revue fussent bien présentés. Et, si au cours de sa longue carrière, il eut à supporter de cruelles déceptions, il put, comme directeur de la Resue suisse de zoologie constater avec une grande satisfaction la réussite d'une œuvre qu'il avait entreprise avec un beau courage, avec confiance et enthousiasme malgré les avertissements, les conseils et le pessimisme qui l'entouraient. Ses efforts furent récompensés; la Revue suisse de zoologie était devenue l'organe indispensable aux zoologistes de notre pays.

Maurice Bedot avait pris une part très active à la constitution de la Société zoologique suisse, il étudia avec M. le professeur Th. Studer un projet de statuts qui fut adopté en 1894 dans une réunion à Schaffhouse. Il en fut le secrétaire pendant plusieurs années. La Société zoologique suisse reconnaissant dans son assemblée générale de Berne, en 1898, les grands services que rendait la Revue décida de demander au Conseil fédéral un crédit. Ce crédit fut accordé, renouvelé chaque année et même augmenté. Les autorités municipales soulignèrent aussi leur admiration pour l'œuvre de Maurice Bedot et la confiance qu'elles lui témoignaient en confirmant, en 1906, par décision du Conseil administratif, l'appui qu'elles s'étaient engagées à donner à la Revue au début de son existence. 
C'est la place ici de rappeler que Maurice Bedot fut le secrétaire général du $6^{\text {me }}$ congrès international de zoologie, tenu à Berne du 14 au 19 août 1904. Grâce à son énergie, le Compte rendu des séances, un beau volume de plus de 700 pages sortait de presse au mois de mai déjà de l'année suivante.

Pour donner une idée complète de toutes les initiatives que prit Maurice Bedot dans le domaine des publications nous mentionnerons celles qu'il fit paraître sous les auspices du Muséum d'histoire naturelle et grâce encore à la générosité des Conseils de la Ville. Le Catalogue illustré de la collection Lamarck dont la première partie concernant les fossiles parue en 1918, a pour but de faire connaître les exemplaires types appartenant au $\mathrm{Mu}$ séum, décrits par l'illustre naturaliste. Ces exemplaires étant trop précieux pour pouvoir être communiqués aux spécialistes étrangers désireux de les comparer avec d'autres spécimens, il s'agissait de les représenter par des reproductions photographiques aussi fidèles que possible. Ce catalogue composé de 117 planches très belles, in- $4^{\circ}$ est accompagné des diagnoses de Lamarck et d'une notice critique de J. Favre. Un Catalogue général des minéraux, destiné à servir de guide dans la collection minéralogique par E. Joukowsky, une brochure de F. de Schæck sur les Oiseaux du port de Genèse en hiver, ornée de 46 figures dans le texte, donne la description des Oiseaux de passage qui viennent de plus en plus nombreux séjourner dans notre port pendant l'hiver et d'intéressants renseignements sur leurs mœurs.

Enfin citons le Catalogue des Invertébrés de la Suisse, dont déjà dix-sept fascicules ont été publiés. Dans la préface du premier fascicule, Maurice Bedot explique qu'il estime urgent de dresser l'inventaire des connaissances que l'on a actuellement sur tous les groupes d'Invertébrés de la Suisse; les travaux faunistiques qui les concernent sont disséminés dans de nombreuses revues et périodiques ce qui rend toujours plus difficile leur étude. Ces "catalogues" devaient comprendre l'énumération et les diagnoses de toutes les espèces décrites à ce jour avec les indications sur leur habitat et la bibliographie complète. Comme il a été dit plus haut, dix-sept groupes d'Invertébrés ont été traités de la sorte par 11 auteurs différents et la Suisse possède une œuvre faunistique digne de celles qui sont publiées maintenant par les grands pays voisins. Maurice Bedot ajoutait dans sa préface que ce travail 
devait forcément présenter des lacunes, car de nombreux groupes d'Invertébrés n'ont pas encore été étudiés d'une façon approfondie dans notre pays, mais qu'il estimait "rendre service aux zoologistes en leur indiquant non seulement ce qui a été décrit jusqu'à présent, mais surtout ce qu'il faut encore chercher ".

Nous voulons terminer sur cette citation qui nous montre Maurice Bedot, sachant ce que la direction d'une publication représente de soucis, de travail ingrat, d'abnégation, entreprenant néanmoins cette œuvre nouvelle afin de "rendre service».

Ouvrier modeste et consciencieux que la vanité n'a jamais atteint un instant, il a cherché toute sa vie à rendre service. Ce fut un bon serviteur de sa patrie et de la science. 


\section{PUBLICATIONS DE MAURICE BEDOT}

1. Benot, M. Sur la faune des Siphonophores du Golfe de Naples. Mitth. zool. Stat. Neapel, Bd. 3, Heft 1-2 (1881), pp. 121-123. Leipzig, 1882, $8^{\circ}$.

2. - Recherches sur le foie des Vélelles. C.: R. Acad. Sc., Tome 98, pp. 1004-1006. Paris, 1884, $4^{0}$.

3. - Recherches sur le développement des nerfs spinaux chez les Tritons. Arch. Sc. phys. nat., (3) Tome 11, pp. 117-145, pl. 1. Genève, 1884, 8º - Et in: Rec. zool. suisse, Tome 1, pl. 9, pp. 161-188. Genève, 1884, $8^{\circ}$.

4. - Recherches sur les Vélelles. Ex.: C. R. Soc. Phys. Hist. Nat. Genève. In.: Arch. Sc. phys. nat., (3) Tome 11, pp. 328-330. Genève, $1884,8^{\circ}$.

5. - Recherches sur l'organe central et le système vasculaire des Vélelles. (Mémoire couronné par l'Université de Genève, Prix Davy). Rec. zool. suisse, Tome 1, pp. 491-517, pl. 25 et 26. Genève, 1884, $8^{\circ}$.

6. - Sur l'histologie de la Porpita mediterranea. Rec. zool. suisse, Tome 2, pp. 189-194. Genève, 1885, $8^{\circ}$.

7. - Contribution à l'étude des Vélelles. Rec. zool. suisse, Tome, 2, pp. 237-251, pl. 9. Genève, $1885,8^{\circ}$.

8. - Recherches sur les cellules urticantes. Rec. zool. suisse, Tome 4, no 1 (1886), pp. 51-70, pl. 2 et 3. Genève, 1888, $8^{\circ}$.

9. - Sur l'A galma clausi n. sp. Rec. zool. suisse, Tome 5, no 1 (1888), pp. 73-91, pl. 3 et 4 . Genève, 1892, $8^{\circ}$.

10. - Procédé de conservation des animaux marins inférieurs. Ex: C. R. Soc. Phys. Hist. nat. Genève. In: Arch. Sc. phys. nat., (3) Tome 21, pp. 556-558. Genève, 1889, $8^{\circ}$.

11. - Observations sur les nématocystes. Ex: C. R. Soc. Phys. Hist. nat. Genève. In: Arch. Sc. phys. nat., (3) Tome 22, pp. 606-608. Genève, $1889,8^{\circ}$.

12. - Bathyphysa grimaldii $n$. sp. Siphonophore bathypélagique de l'Atlantique nord. Résultats des campagnes scientifiques du Prince de Monaco, fasc. 5, 11 pp., 1 pl. Monaco, 1893, $4^{\circ}$.

13. - Camille Pictet. Rev. suisse Zool., Tome 1, pp. I-IV. Genève, $1893,8^{\circ}$.

14. - Revision de la famille des Forskalidae. Rev. suisse Zool. Tome 1, pp. 231-254. Genève, 1893, 8º.

15. - et C. Pictet. Compte rendu d'un voyage scientifique dans l'Archipel malais. Genève, 1893, $8^{\circ}$.

16. - Hermann Fol, sa vie et ses travaux. Rev. suisse Zool., Tome 2, pp. 1-21, 1 portrait. Genève, 1894, $8^{\circ}$.

17. - Note sur une larve de Vélelle. Rev. suisse Zool., Tome 2, pp. 463-466, pl. 21. Genève, 1894, 80. 
18. - Notes anthropologiques sur le Valais. Bull. Soc. Anthropol. Paris, (4) Tome 6, pp. 486-495. Paris, 1895, $8^{\circ}$.

19. - Les Siphonophores de la baie d'Amboine, étude suivie d'une revivision de la famille des Agalmidae. Rev. suisse Zool., Tome 3, no 3, pp. 367-414, pl. 12. Genève, 1896, $8^{\circ}$.

20. - Note sur les cellules urticantes. Rev. suisse Zool., Tome 3, no 4, pp. 533-539, pl. 18. Genève, 1896, $8^{\circ}$.

21. - Recherches sur la population du Valais. Ex: C. R. Soc. Phys. Hist. nat. Genève. In: Arch. Sc. phys. nat., (4) Tome 6, pp. 302-303. Genève, 1898, $8^{\circ}$.

22. - Notes anthropologiques sur le Valais, II. Bull. Soc. Anthropol., Paris, (4) Tome 9, pp. 222-236. Paris, 1898, $8^{\circ}$.

23. - et A. Cartier. Notice sur le Musée d'histoire naturelle de Genève. Genève, 1899, $8^{0}$.

24. - et C. Pictet. Hydraires provenant des campagnes de l'Hirondelle (1886-1888). Résultats des campagnes scientifiques du Prince de Monaco, fasc. 18, 59 pp., 10 pl. Monaco, 1900, $4^{\circ}$.

25. - Matériaux pour servir à l'histoire des Hydroüdes, 1 re période. Rev. suisse de Zool., Tome 9, pp. 379-515. Genève, 1901, 80.

26. - Nouvelles recherches sur la Bathyphysa grimaldii. Ex: C. R. Soc. Phys. Hist. nat. Genève. In: Arch. Sc. phys. nat., (4) Tome 15, pp. 464-465. Genève, 1903, $8^{\circ}$.

27. - Siphonophores provenant des campagnes du Yacht PrincesseAlice (1892-1902). Résultats des campagnes scientifiques du Prince de Monaco, fasc. 27, 29, pp., 4 pl. Monaco, 1904, $4^{\circ}$.

28. - Matériaux pour servir à l'histoire des Hydroüdes, 2 me période (18211850). Rev. suisse de Zool., Tome 13, pp. 1-183. Genève, 1905, $8^{\circ}$.

29. - Henri de Saussure, notice biographique. Rev. suisse de Zool., Tome 14, pp. 1-32, 1 portrait. Genève, 1906, $8^{\circ}$.

30. - Madréporaires d'Amboine. Rev. suisse Zool., Tome 15, pp. 143292, pl. 5-50. Genève, 1907, $8^{\circ}$.

31. - Sur un animal pélagique de la région antarctique. Expédition antarctique française (1903-1905), commandée par le $D^{r}$ Jean Charcot, 5 pp., 1 pl. Paris, 1908, $4^{\circ}$.

32. - La faune eupélagique (Holoplancton) de la baie d'Amboine et ses relations avec celle des autres océans. Rev. suisse Zool., Tome 17, pp. 121-142. Genève, 1909, $8^{\circ}$.

33. - Sur la faune de l'Archipel malais (résumé). Rev. suisse Zool., Tome 17, pp. 143-169. Genève, 1909, $8^{\circ}$.

34. - Matériaux pour servir à l'histoire des Hydrö̈des, 3me période (1851-1871). Rev. suisse Zool., Tome 18, p.p. 189-490. Genève, $1910,8^{0}$.

35. - Notes sur les Hydroüdes de Roscoff. Arch. Zool. expér., (5) Vol. 6., pp. 201-208, pl. 11. Paris 1911, 80.

36. - Sur la nomenclature des Hydres. Zool. Anz., Bd. 39, pp. $602-$ 604. Leipzig, 1912, $8^{\circ}$. 
37. - Matériaux pour servir à l'histoire des Hydroüdes, $4^{\mathrm{me}}$ période, (1872-1880). Rev. suisse Zool., Tome 20, pp. 213-469. Genève, $1912,8^{\circ}$.

38. - Nouvelles notes sur les Hydroüdes de Roscoff. Arch. Zool. expér., Vol. 54, fasc. 3 (1914), p. 79-98, pl. 5. Paris, 1914-15, 80.

39. - A propos d'Antenella simplex. Arch. Zool. expér., Vol. 54, Notes et Revues, no 5 (1914), p. 120. Paris, 1914-15, $8^{\circ}$.

40. - Sur la variation des caractères spécifiques chez les Némertésies. Bull. Inst. océanographique Monaco, no 314, 8 pp., fig. Monaco, $1916,8^{\circ}$.

41. - Matériaux pour servir à l'histoire des Hydroüdes, $5^{\mathrm{me}}$ période (18811890). Rev. suisse Zool., Tome 24, pp. 1-349. Genève, 1916, $8^{\circ}$.

42. - Sur le genre Kirchenpaueria. Rev. suisse Zool., Tome 24, pp. 637-648. Genève, 1916, 8 .

43. - Le genre Nemertesia. Mém. Soc. Phys. Hist. nat. Genève, Vol. 39, fasc. 1, 52 pp., Genève, 1917, $4^{\circ}$.

44. - Le genre Antennella. Rev. suisse Zool., Tome 25, pp. 111-129. Genève, 1917, $8^{\circ}$.

45. - Matériaux pour servir à l'histoire des Hydroüdes, $6^{\text {me }}$ periode (1891-1900). Rev. suisse Zool., Tome 26, fasc. supplémentaire, 376 pp. Genève, 1918, 8 .

46. - Essai sur l'évolution du règne animal et la formation de la société. Genève et Paris, 1918, $8^{\circ}$.

47. - Le développement des colonies d'Aglaophenia. C. R. Soc. Phys. Hist. nat. Genève, Vol. 36, no 2, pp. 50-57, figg. Genève, 1919, $8^{\circ}$.

48. - Les variations d'Aglaophenia pluma (L.). Rev. suisse Zool. Tome 27, no 7, pp. 243-281, figg. Genève, 1919, $8^{\circ}$.

49. - Edmond Béraneck, 1859-1920. Rev. suisse Zool., Tome 28, no 10 (1920). Genève, 1921, $8^{\circ}$.

50. - Notes systématiques sur les Plumularides, $1^{\mathrm{re}}$ partie. Rev. suisse Zool., Tome 28, no 15 (1921), pp. 311-356. Genève, 1921, $8^{\circ}$.

51. - Notes systématiques sur les Plumularides, $2^{\mathrm{me}}$ partie. Rev. suisse Zool., Tome 29, no 1 (1921), pp. 1-40. Genève, So.

52. - Hydrö̈des provenant des campagnes des yachts Hirondelle et Princesse-Alice (188\%-1912). I. Plumularidae. Résultats des campagnes scientifiques du Prince de Monaco, fasc. 60, 74 pp., 6 pl. Monaco, $1921,4^{\circ}$.

53. - Les caractères sexuels secondaires des Plumularides. Rev. suisse Zool., Tome 29, no 4 (1922), pp. 147-166. Genève, 1922, $8^{0}$.

54. - Les Musées. Leur utilité et leurs défauts. Genève, 1922, $8^{\circ}$.

55. - Notes systématiques sur les Plumularides. ${ }^{\mathrm{me}}$ partie. Rev. suisse Zool., Tome 30, no 7 (1923), pp. 213-243, figg. Genève, 1923, $8^{\circ}$.

56. - Matériaux pour servir à l'histoire des Hydrö̈des, '̧me période (1901 à 1910). Rev. suisse Zool., Tome 32, fasc. supplémentaire, 657 pp. Genève, $1925,8^{\circ}$. 


\section{$2 \mathrm{BHL}$ Biodiversity Heritage Library}

Revilliod, P. 1928. "Maurice Bedot 1859-1927." Revue suisse de zoologie 35, 1-16. https://doi.org/10.5962/bhl.part.117615.

View This Item Online: https://www.biodiversitylibrary.org/item/148556

DOI: https://doi.org/10.5962/bhl.part.117615

Permalink: https://www.biodiversitylibrary.org/partpdf/117615

\section{Holding Institution}

American Museum of Natural History Library

\section{Sponsored by}

BHL-SIL-FEDLINK

\section{Copyright \& Reuse}

Copyright Status: In copyright. Digitized with the permission of the rights holder.

Rights Holder: Muséum d'histoire naturelle - Ville de Genève

This document was created from content at the Biodiversity Heritage Library, the world's largest open access digital library for biodiversity literature and archives. Visit BHL at https://www.biodiversitylibrary.org. 